\title{
HIGH INTENSITY TRAINING AND TREADMILL SPRINT PERFORMANCE
}

\author{
M. E. CHEETHAM, BSc and C. WILLIAMS, BSc, MSc, PhD \\ Department of Physical Education and Sports Science, Loughborough University of Technology
}

\begin{abstract}
Twelve county standard hockey players completed a 30 second sprint on a non-motorised treadmill and an uphill treadmill running test to determine maximum oxygen uptake $\left(\mathrm{V}_{2} \mathrm{max}\right)$ before and after 6 weeks of high intensity training (fast runs 3-5 miles, intervals $30-300 \mathrm{~m}$ and circuit training), whilst 11 club standard players completed the same tests without any additional training. For the county standard group there was an $11.1 \%$ and $5.0 \%$ improvement in peak running speed and distance covered on the sprint treadmill respectively, a $4.2 \%$ improvement in $\mathrm{VO}_{2}$ max and an $11.5 \%$ improvement in run time to exhaustion during the $\mathrm{V}_{2}$ max test (all $p<0.01$ ). No changes were observed for the club standard group. There were large increases in blood lactate (county group $13.26 \pm 1.83 \mathrm{mM}$ ) and blood glucose (county group $1.56 \pm 0.71 \mathrm{mM}$ ) concentrations as a result of the treadmill sprint, but there were no additional changes in these variables as a result of training. Thus, the mechanism of adaptation in this type of brief maximal exercise remains in question.
\end{abstract}

Key words: Training, Sprinting, Metabolism, Testing

\section{INTRODUCTION}

The causes of fatigue and the factors which limit performance in sprint running exercise are not fully understood. One means of studying the factors which limit performance is to examine the responses to training and the metabolic changes which accompany training-induced improvements in performance. Several studies have demonstrated improvements in the ability to perform high intensity exercise after various types of training. Extended run times to exhaustion during uphill treadmill running have been found after both general conditioning (Cunningham and Faulkner, 1969) and interval training (Houston and Thomson, 1977), whilst an increased power output during maximal cycling tests has been shown following sprint training (Weltman et al, 1978).

Uphill treadmill running and sprint cycling tests have generally been used as the exercise tasks during these studies, because the performance improvement can be described either in terms of the increased run times to exhaustion during treadmill running, or by the increased power output during sprint cycling. However, sprint running has been less frequently examined in the laboratory because of methodological limitations. The purpose of the present study was to examine the effect of short term high intensity training on sprint running performance as measured by a new laboratory based test (Lakomy, 1984; Cheetham et al, 1985), and also to examine the metabolic responses to this type of brief maximal exercise before and after training.

\section{METHODS}

\section{Subjects}

Two groups of hockey players participated in this experiment. Twelve female county standard hockey players whose mean ( \pm SD) height, weight and age were 162.5 $(5.7) \mathrm{cm}, 61.0(5.6) \mathrm{kg}$ and $21.3(2.1) \mathrm{yrs}$, agreed to complete 6 weeks of high intensity training which included 4-5 training sessions per week (two fast runs between 3-5 miles, two interval sessions which involved repeated runs of $30-300 \mathrm{~m}$ and at least 1 circuit training session) in addition to their

Address for correspondence:

Miss M. E. Cheetham

Dept. of Physical Education and Sports Science

Loughborough University of Technology

LOUGHBOROUGH

Leics. LE11 3TU normal hockey commitments of 1-2 skill/tactics sessions and 1-2 matches per week. A second group of club standard hockey players of height, weight and age $165.2(4.6) \mathrm{cm}$, $61.9(4.4) \mathrm{kg}$ and 21.0 (1.4)yrs volunteered to perform the same laboratory tests as the county standard group, but to continue only with their normal hockey programme of 1-2 matches and one practice session per week.

\section{Protocol}

A non-motorised treadmill was used for the sprint test as this allowed the subjects to run at their own speed and change speed as fatigue occurred. The subjects were thoroughly familiarised with treadmill sprinting in two practice sessions several days before the experiment commenced. On the day of the experiment the subjects arrived at the laboratory after an overnight fast and, following a standardised cycle ergometer warm-up, a 30 second sprint was completed on the non-motorised treadmill from a rolling start. The subjects were asked to run maximally from the start of the test and were verbally encouraged throughout. The sprint test was performed at approximately the same time of the morning before and after training, whilst the post-training tests commenced 2-3 days after the final training session.

The treadmill was interfaced to a microcomputer which continuously monitored the treadmill belt speed and hence recorded the distance which the subjects moved the treadmill belt during the test. The modifications made to the treadmill, the test procedure and reliability have been described previously (Lakomy, 1984; Cheetham et al, 1985).

Capillary blood samples were taken after the standardised warm-up and at 5 minutes following the sprint and were deproteinised, frozen and analysed at a later date for blood lactate and glucose concentrations according to the method described by Maughan (1982). The same procedures were repeated after training.

In addition, an uphill treadmill running test (Taylor et al, 1955) was completed before and after training to determine the maximum oxygen uptake $\left(\mathrm{VO}_{2}\right.$ max $)$ of each individual.

A paired t-test was used to examine differences between the pre- and post-training means.

\section{RESULTS}

The results of the treadmill sprint test for the county standard players are illustrated in Fig. 1 which shows that there was an improvement in running speed throughout the 


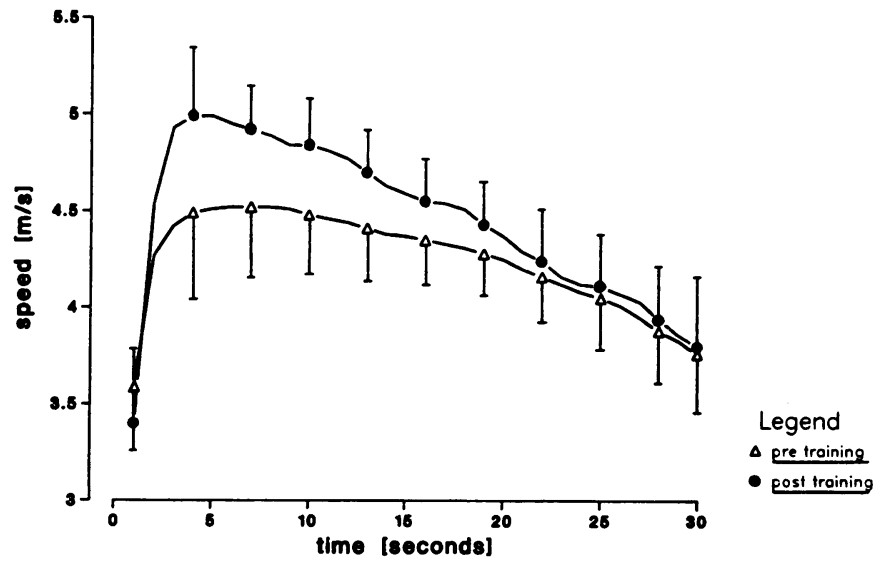

Fig. 1: Running speed during the 30 second sprint on the non-motorised treadmill for the county standard group $(n=12)$ before and after training.

time period of the test after the 6 week period of training in addition to normal hockey activities. The numerical results of the performance tests are shown in Table I. There was an $11.1 \%$ and $5 \%$ improvement in peak running speed and distance covered respectively on the sprint treadmill, a $4.2 \%$ improvement in $\mathrm{VO}_{2}$ max and an $11.5 \%$ improvement in run time to exhaustion during the $\dot{\mathrm{V}}_{2}$ max test (all $\mathrm{p}<$ $0.01)$. Fatigue, during the treadmill sprint test (expressed as the difference between maximum and minimum speeds as a percentage of the maximum speed) increased in absolute terms $(p<0.05)$. There were no changes in any of these variables for club standard group who continued with their normal hockey programme over the 6 week period, but who performed no additional training.

TABLEI

Physiological, and performance characteristics of the county standard and club standard groups before and after 6 weeks' training (mean \pm SD)

\begin{tabular}{|c|c|c|c|c|c|}
\hline & $\begin{array}{c}\mathrm{VO}_{2} \mathrm{MAX} \\
\mathrm{VO}_{2} \max \\
\left(\mathrm{ml}^{\prime} \mathrm{kg}^{-1} \mathrm{~min}^{-1}\right)\end{array}$ & $\begin{array}{l}\text { run } \\
\text { time } \\
\text { (min) }\end{array}$ & $\begin{array}{l}\text { TREAD } \\
\text { peak } \\
\text { speed } \\
\left(\mathrm{m} . \mathrm{sec}^{-1}\right)\end{array}$ & $\begin{array}{l}\text { IILL SPRINT } \\
\text { distance } \\
\text { covered } \\
\text { (m) }\end{array}$ & $\begin{array}{l}\text { EST } \\
\text { fatigue } \\
(\%)\end{array}$ \\
\hline \multicolumn{6}{|c|}{ County Group } \\
\hline \multirow[t]{2}{*}{ Before tr. } & 50.1 & 8.37 & 4.58 & 127.47 & 17.49 \\
\hline & \pm 4.1 & \pm 1.74 & \pm 0.37 & \pm 7.41 & \pm 8.30 \\
\hline \multirow[t]{2}{*}{ After tr. } & $52.2^{* *}$ & $9.33^{* *}$ & $5.09 * *$ & $133.83^{* *}$ & $25.71^{*}$ \\
\hline & \pm 3.7 & \pm 1.71 & \pm 0.34 & \pm 5.97 & \pm 5.80 \\
\hline \multicolumn{6}{|c|}{ Club Group } \\
\hline \multirow[t]{2}{*}{ Before tr. } & 43.9 & 5.45 & 4.27 & 115.41 & 22.03 \\
\hline & \pm 2.5 & \pm 1.04 & \pm 0.38 & \pm 7.99 & \pm 7.37 \\
\hline \multirow[t]{2}{*}{ After $t r}$. & 44.9 & 5.49 & 4.23 & 117.78 & 16.90 \\
\hline & \pm 2.7 & \pm 1.08 & \pm 0.36 & \pm 8.92 & \pm 6.32 \\
\hline
\end{tabular}

\footnotetext{
* $p<0.05{ }^{* *} p<0.01$ significant difference between means before and after training
}

The blood lactate and blood glucose concentrations post warm-up and at 5 minutes after the treadmill sprint before and after training are shown in Table II. Blood lactate concentration after the warm-up and at 5 minutes postexercise was lower for the club standard group after the 6 week period. However, there were no differences in the increase in blood lactate or blood glucose concentrations for either group after training (Fig. 2).

Prior to training those individuals covering the greatest
TABLE \|

Blood lactate and blood glucose concentrations post warm-up and at 5 minutes after the treadmill sprint before and after training (mean \pm SD)

\begin{tabular}{lcccr}
\hline & $\begin{array}{c}\text { Blood lactate } \\
\text { post } \\
\text { warm-up }\end{array}$ & $\begin{array}{c}5^{\prime} \\
\text { post-ex }\end{array}$ & $\begin{array}{c}\text { Blood glucose } \\
\text { post } \\
\text { warm-up }\end{array}$ & $\begin{array}{c}\mathbf{m}^{\prime} \\
\text { post-ex }\end{array}$ \\
\hline County Group & & & & \\
Before tr. & 2.09 & 15.35 & 4.74 & 6.19 \\
& \pm 1.30 & \pm 2.17 & \pm 0.28 & \pm 0.73 \\
After tr. & 1.55 & 14.69 & 4.75 & 6.22 \\
& \pm 0.61 & \pm 2.11 & \pm 0.67 & \pm 0.48 \\
\hline Club Group & & & & \\
Before tr. & 2.82 & 14.86 & 4.31 & 5.61 \\
& \pm 0.93 & \pm 1.67 & \pm 0.39 & \pm 0.70 \\
After tr. & $2.26^{*}$ & $13.78^{* *}$ & 4.46 & 5.61 \\
& \pm 0.75 & \pm 1.57 & \pm 0.30 & \pm 0.22 \\
\hline
\end{tabular}

* $p<0.05 * * p<0.01$ significant difference between means, before and after training

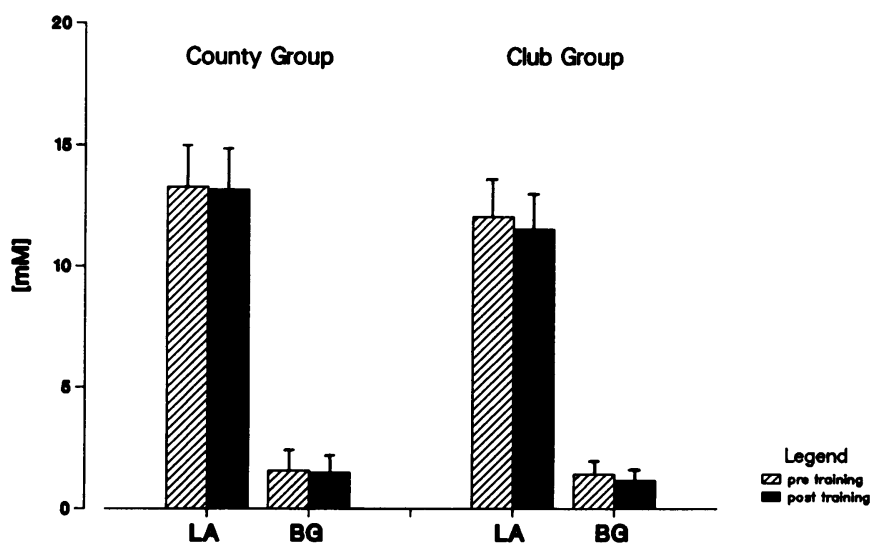

Fig. 2: Changes in blood lactate (BL) and blood glucose (BG) concentrations as a result of the 30 second sprint for the county $(n=12)$ and club standard $(n=11)$ groups before and after training.

distance on the treadmill during the $\mathbf{3 0}$ second sprint also had the highest blood lactate concentration at 5 minutes after the sprint $(r=0.67, p<0.01 ; n=23)$. A relationship was also found between these two variables for the county standard group only, both before and after training $(r=0.73$

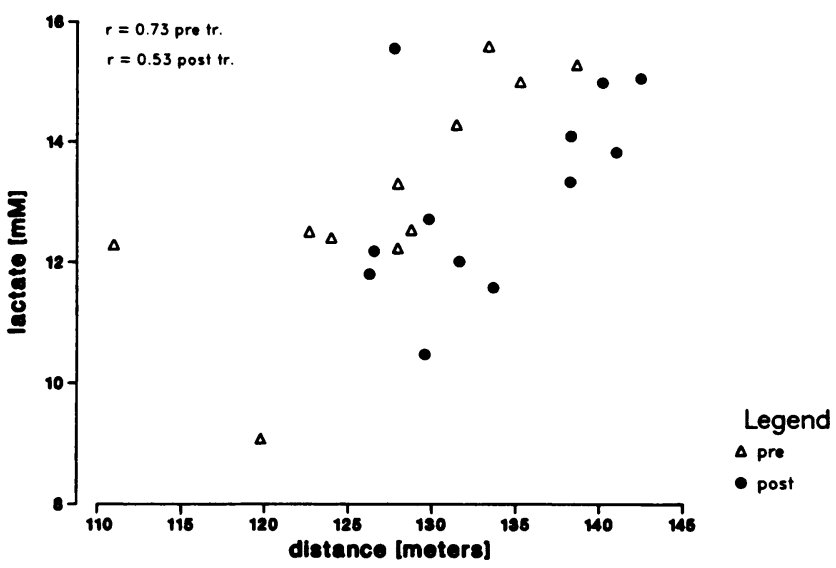

Fig. 3: Relationship between distance run during the 30 second sprint and blood lactate concentration after 5 minutes of passive recovery before and after training for the county standard group ( $n=12$ ). 
$p<0.01$ and $r=0.53 p<0.05$ pre- and post-training respectively) (Fig. 3).

However, no relationship was found between $\mathrm{VO}_{2}$ max and peak running speed on the sprint treadmill ( $r=0.34$ and $r=0.02$ pre- and post-training respectively) or between run time during the $\mathrm{VO}_{2}$ max test and distance covered on the sprint treadmill $(r=0.37$ and $r=-0.04$ pre- and post-training respectively).

\section{DISCUSSION}

The improvements gained in peak running speed and distance covered on the sprint treadmill are of a similar magnitude to those improvements found in other studies where the exercise test used to monitor the training response was performed at maximal rates (Weltman et al, 1978; Campbell et al, 1979; Belcastro et al, 1981). However, all of these studies used sprint cycling as the performance test and blood metabolites were not measured. Those investigations which have examined the metabolic responses to high intensity exercise have used uphill treadmill running as a measure of performance. Both Cunningham and Faulkner (1969) and Houston and Thomson (1977) found increases in the post-exercise blood lactate concentrations of $17 \%$ and $14 \%$ respectively above the pre-training values, which were accompanied by a $23 \%$ and $17 \%$ increase in the run time to exhaustion during the uphill treadmill running test. These increases in blood lactate concentration have been attributed to an increased contribution from anaerobic glycolysis to ATP resynthesis during the run (Cunningham and Faulkner, 1969). However, these results are difficult to interpret, because of the increase in running time after training.

In the present study the blood lactate response was examined after a bout of exercise performed at maximal rates for the same period of time before and after training. Blood lactate concentration at 5 minutes after this type of exercise has been shown to reflect muscle lactate at cessation of the sprint (Cheetham et al, 1986). Therefore, the significant relationship found between blood lactate concentration following the sprint test and performance during the sprint, both before and after training, would tend to suggest that inter-individual variations in performance may be partially related to the ability to supply energy from anaerobic glycolysis. Similar findings were made by Ohkuwa et al (1984) who found a significant relationship between $400 \mathrm{~m}$ times and blood lactate concentration after the sprint in untrained and endurance trained runners $(r=$ $0.76, p<0.05$ and $r=0.71, p<0.05$ ) but found no significant relationship for a group of sprinters. In the present study the training-induced improvements in peak running speed and distance covered on the sprint treadmill were not accompanied by any increase in blood lactate concentration. However, it has been suggested that endurance training may affect lactate clearance rather than lactate production (Donovan and Brooks, 1983) although these findings have been recently disputed (Favier et al, 1986). If the suggestion of increased removal of lactate is accepted and also applied to interval training, it is possible that the muscle lactate concentration could have been higher after training as there would be little time for increased clearance during the sprint, and that more lactate was removed from the circulation in the recovery period. The results of a recent study examining muscle metabolism before and after sprint training found an improvement of $7.5 \%$ and $8.2 \%$ respectively in peak and mean power output during sprint cycling with a 7\%, but not statistically significant, increase in muscle lactate concentration above the pre-training values (Boobis et al, 1983). Whether or not the contribution from anaerobic glycolysis is increased during this type of exercise, as a result of training, is still in question.

As $\dot{\mathrm{VO}}_{2}$ max was increased the possibility of an increased aerobic contribution to the sprint cannot be ruled out. However, the improvements in $\mathrm{VO}_{2}$ max were relatively small $(4.2 \%)$. This may be because of the well-trained status of the subjects prior to the study. A similar (4.1\%) but not statistically significant, training-induced improvement in $\grave{V}_{2}$ max after high intensity training has been shown for a group of highly trained male subjects, whilst a $4.6 \%$ improvement in $\mathrm{V}_{2}$ max was found for female games players after 6 weeks of endurance training at $90 \% \mathrm{VO}_{2}$ max (Houston and Thomson, 1977; Williams and Nute, 1986). The major improvement in the present study during the $\mathrm{VO}_{2}$ max test was in the $11.5 \%$ increase in run time to exhaustion. It has been suggested, as previously mentioned, that such improvements in run time may be a result of a greater anaerobic contribution to the latter stages of the run rather than to any increase in muscle oxidative capacity (Davies et al, 1982). However, as there was no evidence of an increased lactate concentration after the sprint test an alternative explanation may lie in an improved muscle buffering capacity as has been found recently after sprint training (Sharp et al, 1986). Furthermore, any small changes in muscle oxidative capacity that may have occurred are unlikely to have had a substantial effect on sprint performance. In one of the few studies which has examined the effect of an improved oxidative capacity on sprint performance, no changes in sprint cycling performance were found after a short term endurance training programme which resulted in marked increases in $\dot{\mathrm{VO}}_{2}$ max and in endurance capacity (Hardman et al, 1986). As any small changes in the aerobic contribution to the sprint are unlikely to have influenced performance and the blood lactate response to the sprint was the same before and after training, an improvement in economy (defined as force developed in relation to ATP utilised) may have occurred (Westra et al, 1985). Alternatively, blood lactate concentrations sampled at 5 minutes post-exercise may not adequately reflect any training-induced changes in muscle metabolism.

The very low correlations found between performance during the $\dot{\mathrm{V}}_{2}$ max test and the treadmill sprint test are consistent with the findings of Katch and Weltman (1979) who compared power output during cycle ergometer sprinting and $\mathrm{VO}_{2}$ max as determined by treadmill running. These are not surprising findings when the different metabolic requirements of the two tests are considered. Therefore, the training-induced improvements in both sprint performance and in $\mathrm{VO}_{2}$ max in this study, are more likely to be a result of the general nature of the training programme than to any underlying change in metabolism that affects both $\mathrm{VO}_{2}$ max and sprint performance.

In summary this study has demonstrated that short term high intensity training results in an improvement in the sprint running performance and in the $\dot{\mathrm{VO}}_{2}$ max of previously well trained female games players. The improvements in sprint performance could not be accounted for by any change in the post-exercise blood lactate concentration. Hence, the mechanism of adaptation in this type of brief maximal exercise remains in question. 


\section{References}

Belcastro, A. N., Campbell, C. J., Bonen, A. and Kirby, R. L., 1981 "Adaptation of human skeletal muscle myofibril ATPase activity to power training". Aust.J.Sports Med. 13: 93-97.

Boobis, L. H., Williams, C. and Wootton, S. A., 1983 "Influence of sprint training on muscle metabolism during brief maximal exercise in man". J.Physiol. 342: 36-37P.

Campbell, C. J., Bonen, A., Kirby, R. L. and Belcastro, A. N., 1979 “Muscle fiber composition and performance capacities in women". Med.Sci.Spts 11.3: $260-265$.

Cheetham, M. E., Boobis, L. H., Brooks, S. and Williams, C., 1986 "Human muscle metabolism during sprint running". J.Appl.Physiol. 61 (1): 54-60.

Cheetham, M. E., Williams, C. and Lakomy, H. K. A., 1985 "A laboratory based running test: metabolic responses of sprint and endurance trained athletes". Brit.J.Spts.Med. 19: 81-84.

Cunningham, D. A. and Faulkner, J. A., 1969 "The effects of training on aerobic and anaerobic metabolism during a short exhaustive run". Med.Sci.Sport 1: 65-69.

Davies, K. J. A., Packer, L. and Brooks, G. A., 1982 "Exercise bioenergetics following sprint training". Arch.Biochem.Biophys. 215: 260-265.

Donovan, C. M. and Brooks, G. A., 1983 "Endurance training affects lactate clearance not lactate production". Am.J.Physiol. 244 (7): E83-92.

Favier, R. J., Constable, S. H., Chen, M. and Holloszy, J. O., 1986 "Endurance training reduces lactate production". J.Appl.Physiol. 61: 885-889.

Hardman, A. E., Williams, C. and Wootton, S. A., 1986 "The influence of shortterm endurance training on maximum oxygen uptake, submaximum endurance and the ability to perform brief, maximal exercise". J.Sports Sci. 4: 109-116.

Houston, M. E. and Thomson, J. A., 1977 "The response of enduranceadapted adults to intense anaerobic training". Eur.J.appl.Physiol. 36: 207-213.
Katch, V. L. and Weltman, A., 1979 "Interelationship between anaerobic power output, anaerobic capacity and aerobic power". Ergonomics 22: 325-332.

Lakomy, H. K. A., 1984 "An ergometer for measuring the power generated during sprinting". J.Physiol. 354: 33P.

Maughan, R. J., 1982 "A simple rapid method for the determination of glucose, lactate, pyruvate, alanine, 3- hydroxybutyrate and acetoacetate on a single 20- $\mu$ l blood sample". Clin.Chi.Acta 122: 231-240.

Ohkuwa, T., Yoshinobu, K., Katsumata, K., Nakao, T. and Miyamura, M., 1984 "Blood lactate and glycerol after $400 \mathrm{~m}$ and $3000 \mathrm{~m}$ runs in sprint and long distance runners". Eur.J.Appl.Physiol. 53: 213-218.

Sharp, R. L., Costill, W. J., Fink, D. L. and King, D. S., 1986 "Effects of eight weeks of bicycle ergometer sprint training on human muscle buffer capacity". Int.J.Sports Med. 7: 13-17.

Taylor, H. L., Buskirk, E. and Henschel, A., 1955 "Maximum oxygen intake as an objective measure of cardio-respiratory performance". J.Appl.Physiol. 8: 73-80.

Weltman, A., Moffat, R. J. and Stamford, B. A., 1978 "Supramaximal training in females: Effects on anaerobic power output, anaerobic capacity and aerobic power". J.Sports Med. 18: 237-244.

Westra, H. G., De Hann, A., Van Doorn, J. E.and De Hann, E. J., 1985 "The effect of intensive interval training on the anaerobic power of the rat quadriceps muscle". J.Sports Sci. 3: 139-150.

Williams, C. and Nute, M. G., 1986 "Training-induced changes in endurance capacity of female games players". In: Sports Science (Eds. J. Watkins, T. Reilly and L. Burwitz). Proc. of the VIII Commonwealth and Int.Conf. Sport, Physical Education, Dance, Recreation and Health. Spon Ltd., London pp. 11-17. 